\title{
Diffusion anisotropy descriptor revealing morphology effect of H-ZSM-5 zeolite for olefin catalytic cracking
}

\section{Xiaoliang Liu}

State Key Laboratory of Green Chemical Engineering and Industrial Catalysis, Shanghai Research Institute of Petrochemical Technology, SINOPEC Corp.

\section{Jing Shi}

State Key Laboratory of Green Chemical Engineering and Industrial Catalysis, Shanghai Research Institute of Petrochemical Technology, SINOPEC Corp.

\section{Guang Yang}

State Key Laboratory of Green Chemical Engineering and Industrial Catalysis, Shanghai Research Institute of Petrochemical Technology, SINOPEC Corp.

\section{Jian Zhou}

Shanghai Research Institute of Petrochemical Technology, SINOPEC, Shanghai 201208, P. R. China.

\section{Chuanming Wang}

State Key Laboratory of Green Chemical Engineering and Industrial Catalysis, Shanghai Research Institute of Petrochemical Technology, SINOPEC Corp.

\section{Jiawei Teng}

State Key Laboratory of Green Chemical Engineering and Industrial Catalysis, Shanghai Research Institute of Petrochemical Technology, SINOPEC Corp.

\section{Yangdong Wang}

State Key Laboratory of Green Chemical Engineering and Industrial Catalysis, Shanghai Research Institute of Petrochemical Technology, SINOPEC Corp.

\section{Zaiku Xie ( $\nabla$ xzk@sinopec.com )}

China Petrochemical Corporation (SINOPEC Group)

\section{Article}

Keywords: zeolite catalysis, olefin catalytic cracking, morphology

Posted Date: January 9th, 2021

DOI: https://doi.org/10.21203/rs.3.rs-138171/v1 
License: (c) (i) This work is licensed under a Creative Commons Attribution 4.0 International License. Read Full License

Version of Record: A version of this preprint was published at Communications Chemistry on July 16th, 2021. See the published version at https://doi.org/10.1038/s42004-021-00543-w. 


\title{
Diffusion anisotropy descriptor revealing morphology effect of H-ZSM-5 zeolite for olefin catalytic cracking
}

Xiaoliang $\mathrm{Liu}^{+1}$, Jing $\mathrm{Shi}^{+1}$, Guang Yang ${ }^{1}$, Jian Zhou ${ }^{1}$, Chuanming Wang ${ }^{1}$, Jiawei Teng*1, Yangdong Wang*1 \& Zaiku Xie*2

\begin{abstract}
${ }^{1}$ State Key Laboratory of Green Chemical Engineering and Industrial Catalysis, Shanghai Research Institute of Petrochemical Technology, SINOPEC Corp., Shanghai 201208, China. ${ }^{2}$ China Petrochemical Corporation (SINOPEC Group), Beijing 100728, China. ${ }^{+}$These authors contributed equally: Xiaoliang Liu, Jing Shi. *Correspondence and requests for materials should be addressed to J.T. (email: tengjw.sshy@sinopec.com) or to Y.W. (email: wangyd.sshy@sinopec.com) or to Z.X. (email: xzk@sinopec.com)
\end{abstract}

\begin{abstract}
Zeolite morphology is vital in determining catalytic activity, selectivity and stability in zeolite catalysis, while quantitative description of morphology effect is great challenging but highly desirable. Herein, a descriptor to elucidate the morphology effect is proposed by revealing the diffusion anisotropy in straight and sinusoidal channels of H-ZSM-5 zeolite for olefin catalytic cracking. A series of H-ZSM-5 zeolites with similar nano-sheet morphology were precisely synthesized in which only the length in c-axis varies. It is unexpectedly demonstrated that the catalytic activity and stability can be obviously improved by employing samples with longer length in c-axis. Combining time-resolved in-situ FT-IR spectroscopy with molecular dynamic simulations, we revealed that the difference in catalytic performance can be attributed to the intracrystalline diffusive propensity in different channels. This work not only provides a clear descriptor revealing morphology effect, but also offers deep insight into design of highly effective zeolite catalysts for olefin catalytic cracking.
\end{abstract}

\section{Introduction}

H-ZSM-5 zeolite with MFI topology, has been extensively studied and applied in a series of petrochemical processes due to its abundant well-defined microporous structure and the intrinsic moderate Brønsted acidities ${ }^{1,2}$. The framework of H-ZSM-5 zeolite contains two types of 
intersecting 10-membered-ring channels ${ }^{3,4}$, as shown in scheme 1a. The straight channels $(5.4 \times$ $5.6 \AA$ A) run along the crystallographic b-axis with exposed facet of [010] and the sinusoidal channels $(5.1 \times 5.4 \AA)$ parallel to the a-axis with exposed facets of [101] and [100]. It was reported that regulating the preferred orientations of the pore systems to crystal planes will cause the variety of the H-ZSM-5 morphologies, thus correspondingly affecting the diffusion resistances $^{5,6}$. Morphology adjustment by reducing the particle size and shape with a controllable $\mathrm{a} / \mathrm{b}$ or $\mathrm{a} / \mathrm{c}$ "aspect ratios" is considered as an efficient route to reduce the diffusion path lengths, increase the accessibility of active sites, and finally promote catalytic activities of H-ZSM-5 samples $^{7-12}$. In particular, constructing H-ZSM-5 catalysts with intergrowths on well-defined crystal facet opening ${ }^{7,12}$ or nanosheet morphology with extremely short thickness along b-axis ${ }^{8,9,11}$, showing remarkably longer lifetime in catalytic reactions. Therefore, controlling the morphology of H-ZSM-5 zeolite is of certain considerable importance for the diffusion and shape-selective catalysis. However, the intrinsic relationship between morphology and catalytic property is obscure due to the lack of descriptors revealing morphology effect.

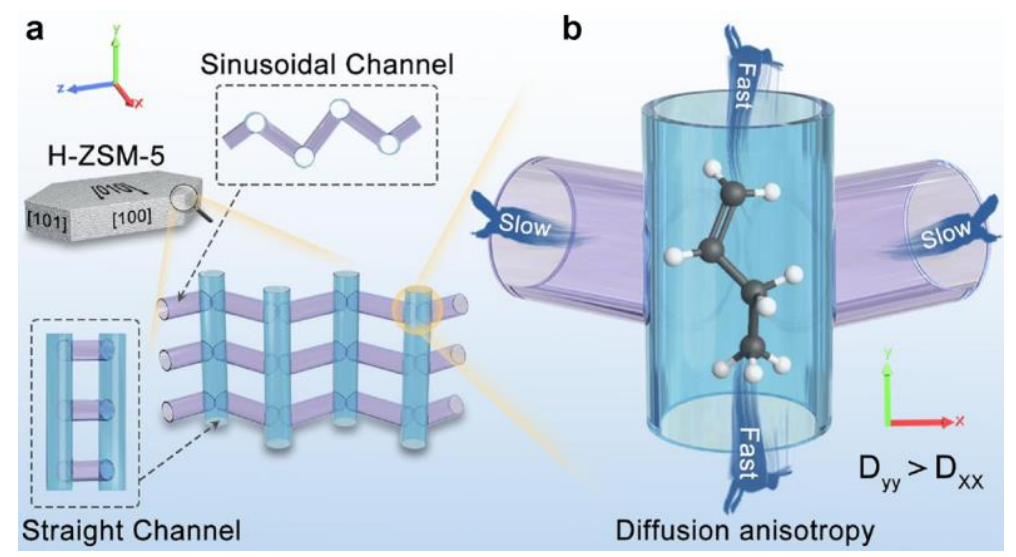

Schame 1 a The framework model of H-ZSM-5 type zeolites composed of two channel directions. The straight and sinusoidal channels running along b-axis and a-axis with similar opening sizes but different geometry shapes. b Schematic view of diffusion anisotropy in two channels network.

To elucidate the key descriptor of morphology effect over H-ZSM-5 zeolite in catalysis process, it is extremely key to differentiate diffusivities in the two-types of pore channels ${ }^{13,14}$. The diffusion in the two-channels system of H-ZSM-5 crystal is actually complicated due to the sensitivity to the kinetic diameter of adsorbed molecules and the topological structures of zeolites. 
The molecules like methanol, xenon, methane etc. are small enough to diffuse randomly through the network of two intersecting channel systems without configuration limitations ${ }^{15-17}$. However, as the size of guest molecules further increasing, such as long chain hydrocarbons $\left(\mathrm{C}_{3}, \mathrm{C}_{4}\right)$ or aromatic molecules, an anisotropic diffusion would occur, where the escape rates of the adsorbate in the intersecting pores of ZSM-5 zeolites are diverse in different channels segments (scheme 1b) $)^{9,15,18,19}$. Therefore, differentiating diffusivities in straight and sinusoidal channels for these anisotropic molecules over H-ZSM-5 zeolites are benefit to the understanding of activity, stability or product distribution in catalysis and the guidance of catalyst design with designated morphology. The catalytic cracking of low-value $\mathrm{C}_{4+}$ olefins fractions (OCC), deriving from fluid catalytic cracking (FCC) and methanol to olefins (MTO) processes ${ }^{7,20-22}$, represents a meaningful route to improve the ethylene and propylene production (important to the polymer industry) using H-ZSM-5 catalysts in industrial application ${ }^{23-27}$. Herein, the olefin catalytic cracking process was employed to clarify the descriptor of morphology effect in catalysis process by revealing the diffusion anisotropy in straight and sinusoidal channels of H-ZSM-5 zeolite.

In this work, several H-ZSM-5 zeolites with controllable nano-sheet morphology were precisely designed: various lengths along c-axis with comparative textures, acidities, lengths of a-axis and thicknesses of b-axis, suggesting equiform diffusion path lengths but different exposed percent of pore channels to defined crystal-facets. A home-made time-resolved in situ FT-IR spectroscopy was developed to study the diffusion properties of zeolites catalysts. Combined with experimental evidences and molecular dynamics simulations, the relationship between morphologies, catalysis behaviors and diffusion properties will be discussed in detail. The key descriptor for the morphology effect in OCC reaction was investigated. For the first time, the diffusion anisotropy behavior is studied by precise morphology adjustment to modulate the catalytic performances.

\section{Results}

\section{Structural characterizations of H-ZSM-5 zeolites.}

The structural characterizations were performed to gain insights into the structures, chemical properties and morphologies of the H-ZSM-5 zeolites. A series of precisely designed H-ZSM-5 samples (denoted as Z-cS, Z-cM and Z-cL) were synthesized and selected for investigation in this 
work. The X-ray diffraction patterns (XRD) in Fig. S1a displays the diffraction peaks of MFI topological structure with considerable crystallographic intensity, indicating a high and similar relative crystallinity over the samples ${ }^{5,28}$. All the Ar physisorption isotherms of the samples show similar shapes (Fig. S1b), indicating a comparable pore structure. The pore volumes, BET surface areas and detailed textural parameters over the samples are provided in Table S1. There are no obvious distinctions in BET surface areas and pore volumes over the selected samples. Brønsted acid sites, as shown at $1545 \mathrm{~cm}^{-1}$ in Fig. S1c, are deemed to be the active sites for olefin catalytic cracking reaction, and their numbers are summarized in Table S1h, in which the total amounts of active sites for various samples are comparative.

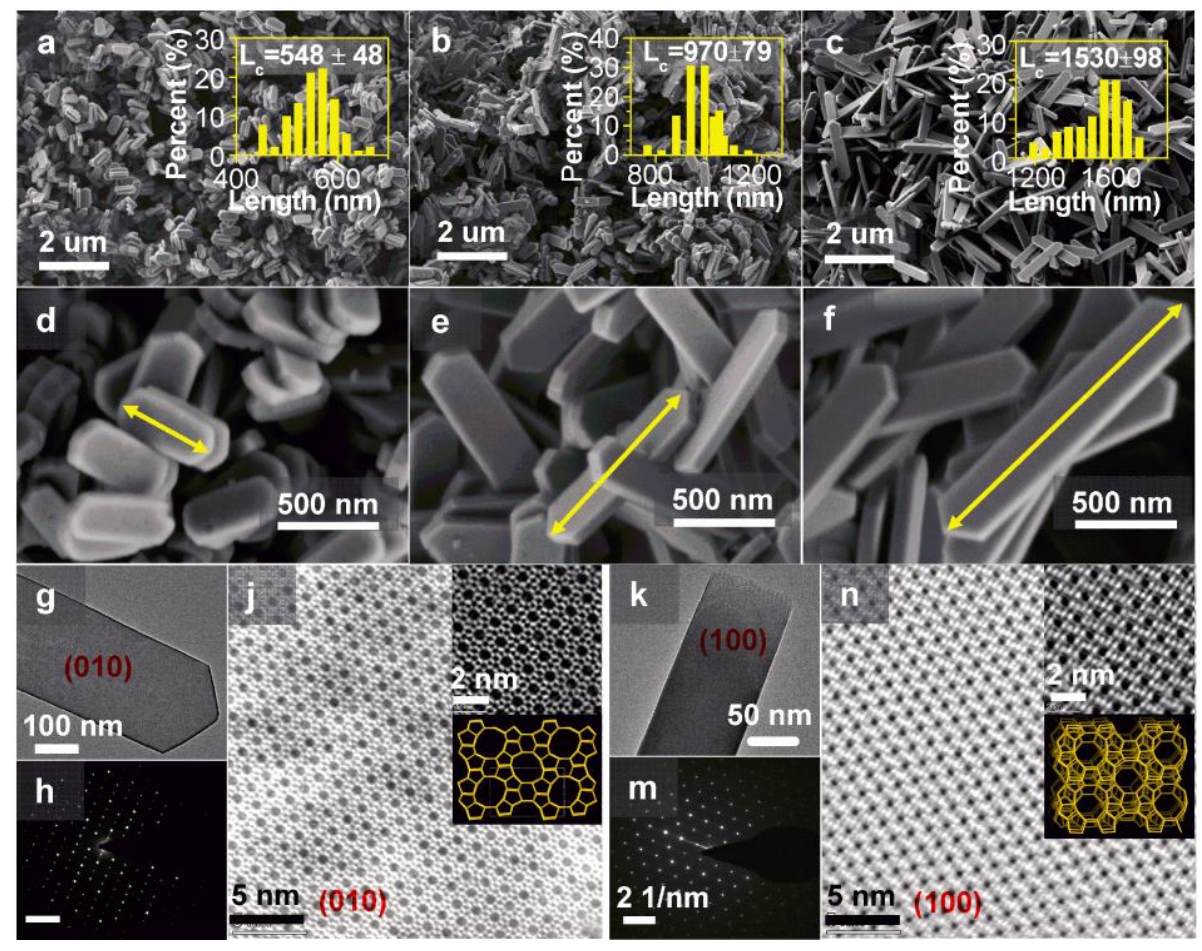

Fig. 1 The morphology of the as-synthesized H-ZSM-5 samples. SEM results of H-ZSM-5 zeolites with similar thicknesses of $\mathrm{a}$ and $\mathrm{b}$ axis but different lengths of $\mathrm{c}$ axis: $\mathbf{a}, \mathbf{d} \mathrm{Z}-\mathrm{cS} ; \mathbf{b}$, $\mathbf{e}$ Z-cM; c, f Z-cL. g TEM image of Z-cL sample with exposed facets of [010] plane. $\mathbf{h}$ The corresponding SAED patterns of [010] plane. $\mathbf{j}$ Aberration-corrected STEM image of Z-cL sample with exposed facets of [010] plane, with the inset on the top right displaying the STEM image with high resolution and the framework structure of H-ZSM-5 projected along [010] plane. $\mathbf{k}, \mathbf{m}$, n TEM image, the corresponding SAED patterns and the aberration-corrected STEM image of Z-cL sample with exposed facets of [100] plane. 
The SEM images of these H-ZSM-5 samples are shown in Fig. 1a-f. All of the samples exhibit a coffin-like nano sheet shape, which is a typical morphology of MFI type zeolites ${ }^{5,28}$. The average lengths of a-, b- and c-axis over corresponding zeolites were counted from Fig. 1a-c by statistics of 100 specimens. As shown in Fig. 1a-f and Fig. S2, the samples present similar lengths of crystal along a- and b-axis, with the average size of $\sim 250 \mathrm{~nm}$ and $\sim 100 \mathrm{~nm}$ respectively, whereas the lengths of c-axis are apparently diverse. Z-cS presents the smallest length of c-axis with a medial of $548 \mathrm{~nm}$, while the mean lengths for Z-cM and Z-cL are $970 \mathrm{~nm}$ and $1530 \mathrm{~nm}$ respectively. According to the TEM and aberration-corrected STEM images, the characteristic 10-membered ring straight channels which run along b-axis can be distinguished along the [010] plane (Fig. 1g-j), and the sinusoidal channels which parallel to a-axis could be observed along the [100] plane (Fig. 1k-n). Besides, the exposure degree of [010] crystal facets were calculated. The results are summarized in Table $1 \mathrm{~b}$, and the detailed calculation procedures are displayed in the methods section. All of these sheet-like zeolites exhibit a dominant exposed plane of [010]. Longer c-axis results in a higher exposed degree of [010] plane and Z-cL presents the highest exposed degree up to $68.9 \%$.

Furthermore, the lattice parameters for the H-ZSM-5 crystal unit cell have been decided from X-ray diffraction methodology, and the diameters of a-, b-, c- directions in one unit cell are considered as $20.07,19.92$, and $13.42 \AA$ in the Pnma space group (orthorhombic), as shown in the databases of IZA structure. The same results were reported in some early papers ${ }^{15,16,18}$. Therefore, the cross section parameters of a unit cell in various crystal facets could be presented. The [010] plane in one unit cell exhibits two pores follow the straight channel path, with a cross section of $20.07 \times 13.42 \AA$. The [100] and [101] direction in one unit cell possess two pores follow the sinusoidal channel path, with a cross sections of $19.92 \times 13.42 \AA$ and $19.92 \times 24.14 \AA$ respectively. Thus, the degree of exposed pore channels per unit area $\left(/ \mathrm{n}^{2}\right)$ in corresponding exposed plane can be calculated, as listed in Table S2. Then, the proportions of the two types of channels in the selected H-ZSM-5 samples can be explicitly computed, as presented in Table 1c and d, and the detailed calculation processes are displayed in Table 1 and methods section. It can be found that as the exposed degree of [010] plane increased, the more percent of straight channels are revealed. 
Table 1 Morphology parameters of the as synthesized H-ZSM-5 samples

\begin{tabular}{|c|c|c|c|c|c|c|}
\hline sample & $\begin{array}{c}\mathbf{L}_{\mathbf{a}}^{\mathbf{a}} \\
{[\mathbf{n m}]}\end{array}$ & $\begin{array}{c}\mathbf{L}_{\mathbf{b}}{ }^{\mathbf{a}} \\
{[\mathrm{nm}]}\end{array}$ & $\begin{array}{c}\mathbf{L}_{\mathbf{c}}{ }^{\mathbf{a}} \\
{[\mathrm{nm}]}\end{array}$ & $\begin{array}{c}{[010]} \\
\text { exposure } \\
\operatorname{degree}^{\mathrm{b}}[\%]\end{array}$ & $\begin{array}{c}\text { Straight } \\
\text { channels } \\
\text { percent }^{\mathrm{c}}[\%]\end{array}$ & $\begin{array}{l}\text { Sinusoidal } \\
\text { channels } \\
\text { percent }^{\mathrm{d}}[\%]\end{array}$ \\
\hline Z-cS & 248 & 100 & 548 & 63.0 & 67.6 & 32.4 \\
\hline Z-cM & 246 & 97 & 970 & 67.2 & 69.7 & 30.3 \\
\hline Z-cL & 249 & 98 & 1530 & 68.9 & 70.4 & 29.6 \\
\hline \multicolumn{7}{|c|}{${ }^{\text {a }}$ Determined by statistics of 100 specimens in SEM images displayed in Fig 1.} \\
\hline \multicolumn{7}{|c|}{${ }^{\mathrm{b}}$ Calculated by the exposure surface areas of various crystal planes. $d_{[010]}=\mathrm{S}_{[010]} /\left(\mathrm{S}_{[010]}+\mathrm{S}_{[100]}+\mathrm{S}_{[101]}\right)$. } \\
\hline \multicolumn{7}{|c|}{${ }^{\mathrm{c}}$ Calculated by the follow expression: $p(\mathrm{str})=.\mathrm{S}_{[010]}{ }^{*} \mathrm{n}_{[010]} /\left(\mathrm{S}_{[010]}{ }^{*} \mathrm{n}_{[010]}+\mathrm{S}_{[100]}{ }^{*} \mathrm{n}_{[100]}+\mathrm{S}_{[101]}{ }^{*} \mathrm{n}_{[101]}\right)$. } \\
\hline \multicolumn{7}{|c|}{${ }^{\mathrm{d}}$ Calculated by the follow expression: $p(\sin )=.100 \%-p($ str. $)$. } \\
\hline \multicolumn{7}{|c|}{ The $\mathrm{n}_{[010]}, \mathrm{n}_{[100]}$ and $\mathrm{n}_{[101]}$ are used to represent the pore amounts per $\mathrm{nm}^{2}$ over corresponding crystal facet. } \\
\hline De & ons fo & $\mathrm{ac}$ & ff expo & ts are d & net & 1.3 \\
\hline
\end{tabular}

Catalytic behaviors over H-ZSM-5 catalysts with various morphology.

In this work, the catalytic behaviors on the cracking of $\mathrm{C}_{4}$ olefin (OCC) were explored over H-ZSM-5 catalysts with various sheet-like morphology, and the results are displayed in Fig. 2. The OCC reactions were performed with a high weight hourly space velocity of pure butene $\left(30 \mathrm{~h}^{-1}\right)$ at $550{ }^{\circ} \mathrm{C}$, as shown in Fig. $2 \mathrm{a}$ and $\mathrm{b}$. In the initial few hours, all of these catalysts presented a relatively stable performance, the $\mathrm{C}_{4}{ }^{=}$conversion and yield of the main products $\mathrm{C}_{2-3}=$ over Z-cL are slightly higher than these over the other two catalysts. As reaction processing, the activities of these catalysts become significantly disparate, with decreased sequence of Z-cL $>$ Z-cM $>$ Z-cS. To directly find out the explicit catalytic distinctions over the catalysts, the detailed $\mathrm{C}_{4}{ }^{=}$ conversions and $\mathrm{C}_{2-3}=$ yields at various reaction time are displayed in Fig. $2 \mathrm{c}$ and d. At initial 10 hours reactions, $\mathrm{C}_{4}=$ conversions are kept above $70 \%$, with lower olefins yields of almost $30 \%$ over all catalysts. The activity of Z-cL is slightly higher than that of other two catalysts. After reacting for 50 hours, Z-cL presents a much slow decreasing tendency, of which $\mathrm{C}_{4}{ }^{=}$conversion and $\mathrm{C}_{2-3}=$ yield could retain $40 \%$ and $18 \%$ after reacting for $50 \mathrm{~h}$, which is obviously higher than 
that of other two catalysts. Generally, the reduction of particle size helps to ensure stability regarding catalyst deactivation for long-term catalytic reactions ${ }^{8-10}$. However, our experimental facts in Fig. 2 unexpectedly point out an obviously opposite result that enlarging the size along c-axis in H-ZSM-5 catalyst can result in a higher OCC catalytic activity and a better stability.
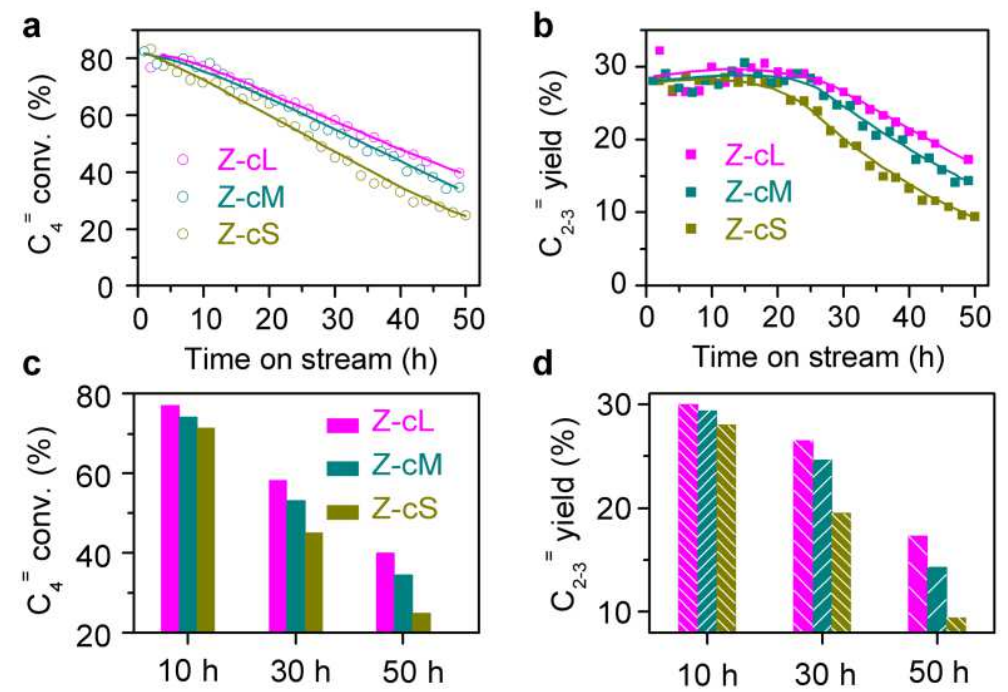

Fig. 2 Catalytic behaviors for $\mathrm{C}_{4}$ olefins catalytic cracking reactions over Z-cS, Z-cM and Z-cL catalysts. a $\mathrm{C}_{4}{ }^{=}$conversions. b Yields of propene and ethene. c, $\mathbf{d}$ detailed $\mathrm{C}_{4}{ }^{=}$conversions and $\mathrm{C}_{2-3}{ }^{2}$ yields at different reaction times. Reaction conditions: $M$ (catalyst) $=0.3 \mathrm{~g} ; P=1.6$ Bar; $T=823 \mathrm{~K} ; F=15 \mathrm{~mL} \mathrm{~h}^{-1}$; Time on stream, $50 \mathrm{~h}$.

It is well known that many factors in H-ZSM-5 crystal can influence the catalysis properties, such as acidities ${ }^{26,29}$, textural structures ${ }^{27}$ and morphology ${ }^{9-12}$. On one hand, the influence of the textural structures and acid sites in our disquisitive catalysts can be excluded due to the similarity in these zeolites, as presented in Fig. S1 and table S1. On the other hand, the characters of morphology are significantly different over those selected catalysts, as shown in Fig. 1 and Table 1, which could be attributed to the probable influencing factor for the unnatural catalytic performance.

\section{Diffusion behaviors of morphology effect over H-ZSM-5 catalysts.}

To obtain better cognition of the relationship between the catalytic properties and the morphology, the uptake rates of $\mathrm{C}_{4}$ molecules (the kinetic diameter is comparable to the pore sizes of H-ZSM-5) over those three samples were compared using our home-made time-resolved in situ 
FTIR spectroscopy. Detailed exploratory operation conditions about this measurement as well as the original uptake curves are shown in Fig. S3 and S4. The diffusion rate $\left(D_{\text {eff }} / L^{2}\right)$ was applied to evaluate the diffusion resistance of these analytic samples, which can be fitted from the normalized uptake curves with Eq. 6 in methods section. Lower $D_{e f f} / L^{2}$ represents a stronger diffusion resistance in zeolite pore systems. Fig. 3a-c displays the normalized uptake curves of $\mathrm{C}_{4}$ molecules adsorption over various zeolites respectively, the uptake rates are increased in the order of Z-cS $<$ Z-cM $<$ Z-cL, indicating that the longer c-axis morphology in H-ZSM-5 leading to the faster diffusion rate. Fig. $3 \mathrm{~d}$ presents the correlation of the diffusivity with the exposure degree of [010] plane over these analytic H-ZSM-5 samples. The diffusivity increases almost linearly with the exposure degree of [010] plane over H-ZSM-5 samples. Meanwhile, the uptake properties of the main product molecule $\left(\mathrm{C}_{3}{ }^{-}\right)$and were performed over the identical samples, As displayed in Fig. S5, the same results of $\mathrm{C}_{3}{ }^{=}$molecule were obtained as that of $\mathrm{C}_{4}{ }^{\overline{ }}$ molecule that enlarging the exposure degree of [010] plane lead to a faster diffusion rate.

Moreover, the diffusion behaviors of Z-cS and Z-cL catalysts for butene molecules were performed with a temperature range of $50-150{ }^{\circ} \mathrm{C}$ (Fig. 3e and f). Here, a lower temperature range was applied for the diffusion measurements since $200{ }^{\circ} \mathrm{C}$ is high enough to cause the polymerization of $\mathrm{C}_{4}{ }^{=}$molecule (Fig. S6). As displayed in Fig. 3g, the apparent diffusive rates over Z-cS are lower than Z-cL at all of the temperatures (detailed fitted data are listed in Table S3), and the activation energy of Z-cS $\left(26.4 \mathrm{~kJ} \mathrm{~mol}^{-1}\right)$ is $30 \%$ higher than that of Z-cL $\left(20.3 \mathrm{~kJ} \mathrm{~mol}^{-1}\right)$. These results further demonstrate the increase of [010] exposure degree in H-ZSM-5 crystal can reduce the diffusion resistance, resulting in a lower active energy barrier to a certain extent. 

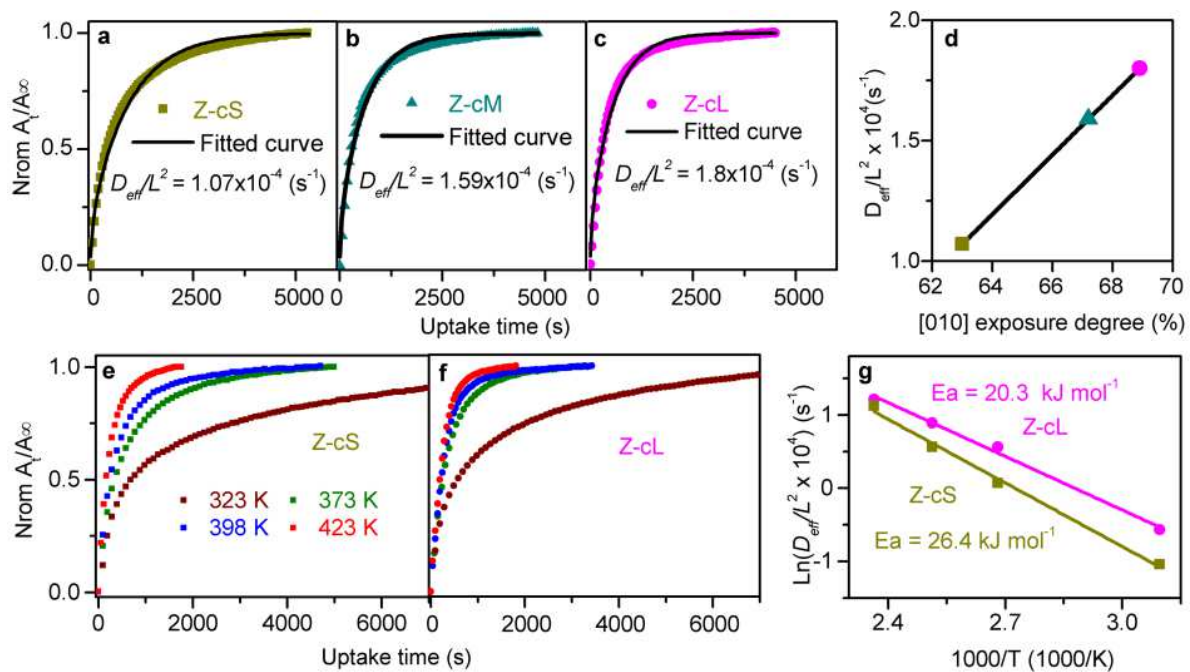

Fig. 3 Diffusion behaviors of $\mathbf{C}_{4}{ }^{=}$in H-ZSM-5 zeolites. Uptake curves of $\mathrm{C}_{4}=$ over H-ZSM-5 samples with different length of c axis: a Z-cS, b Z-cM, c Z-cL. d Correlation of the [010] exposure degrees of H-ZSM-5 zeolites with the diffusion rates fitted by Eq.6. e, $\mathbf{f}$ Uptake curves of $\mathrm{C}_{4}{ }^{=}$over Z-cS and Z-cL with different temperature. $\mathrm{g}$ Arrhenius plot of diffusion rates over Z-cS and Z-cL. The detailed conditions of diffusive measurements are displayed in methods section.

The results presented in Fig. 3 have quantificationally indicated that the exposed degree of crystal-facet orientation is responsible for the various diffusion barriers in H-ZSM-5 crystals. It is worth noting that the diffusivity derived from the time-resolved in situ FTIR spectroscopy is actually the apparent diffusive rates reflecting the combination of surface and intracrystalline diffusion in the nano sheet H-ZSM-5 crystals $^{30,31}$. The surface diffusive mechanism, though remaining unrevealed, is closely related to the external surface characteristics of H-ZSM-5 crystals $^{31,32}$. The intracrystalline diffusion is directly attributed to the properties of nanoporous intracrystalline pore systems ${ }^{33,34}$. Recently, Gao et $\mathrm{al}^{35,36}$ have proposed an approach to directly quantify dual resistance model (DRM) by deducing an approximate expression relying solely on surface permeability from the initial uptake rate $(\alpha / L)$ :

$$
\left.\frac{M_{t}}{M_{\infty}}\right|^{\sqrt{t} \rightarrow 0} \cong \frac{\alpha}{L}(\sqrt{t})^{2}+O\left(\sqrt{t^{3}}\right)
$$

And the intracrystalline diffusivity can be fitted by the following approximation ${ }^{35,37,38}$ : 


$$
\frac{L^{2}}{D_{\text {eff }}}=\frac{L^{2}}{D_{\text {intra }}}+\frac{3 L}{\alpha}
$$

Where $D_{\text {eff }}$ represents the apparent diffusion coefficient, $D_{\text {intra }}$ is the intracrystalline diffusive coefficient, $\alpha$ is the surface permeability, and $L$ is the diffusion path length.

In order to reveal the intrinsic diffusive mechanism of the morphology effect, initial normalized uptake curves presented in Fig. 3a-c were fitted with Eq. 1 and Eq. 2. The fitted curves and the corresponding results of $D_{\text {intra }} / L^{2}$ and $\alpha / L$ are displayed in Fig. 4a-d, respectively. As the enhancement of exposed degree of the [010] plane, similar $\alpha / L$ and apparent increase in $D_{\text {intra }} / L^{2}$ can be observed, which means that the properties of intracrystalline channels instead of the external surface are the decisive factors for the diverse diffusion behaviors of morphology effect. The structural characterizations summarized in Table 1 have demonstrated that the intracrystalline diffusion paths of these three samples are very close due to their similar lengths of a- and b-axis, which are parallel to the diffusion channels, but the percent of straight channels in H-ZSM-5 increases with the enhancement of the exposure degree of [010] plane. Thus, the percent of straight channels were correlated with the intracrystalline diffusivity $\left(D_{\text {intral }} / L^{2}\right)$ over various H-ZSM-5 samples, and the results are presented in Fig. 4e. The intracrystalline diffusivity accelerates with the increasing of the percent of straight channels. When the intracrystalline diffusivity was correlated with the percent of sinusoidal channels, an opposite result can be revealed, as shown in Fig. S7. Therefore, It can be considered that the diffusion rate in straight channels is obviously faster than that in sinusoidal channels for olefins molecules, which can explain the reason why the percent of straight channels accelerating the intracrystalline diffusivity significantly.

According to the results presented above, it can be seen that the diffusion barriers over H-ZSM-5 zeolites are significantly determined by controlling the morphology with various exposed facets degrees after excluding the influence of the textural compositions and active sites. The diffusion anisotropy in intracrystalline channels of H-ZSM-5 crystal is considered to be the essential factor for morphology effect. Due to the preferred diffusive pathway, increasing the straight channels percent by enlarging the [010] plane degree is an efficient route to accelerate the internal diffusive rate. 

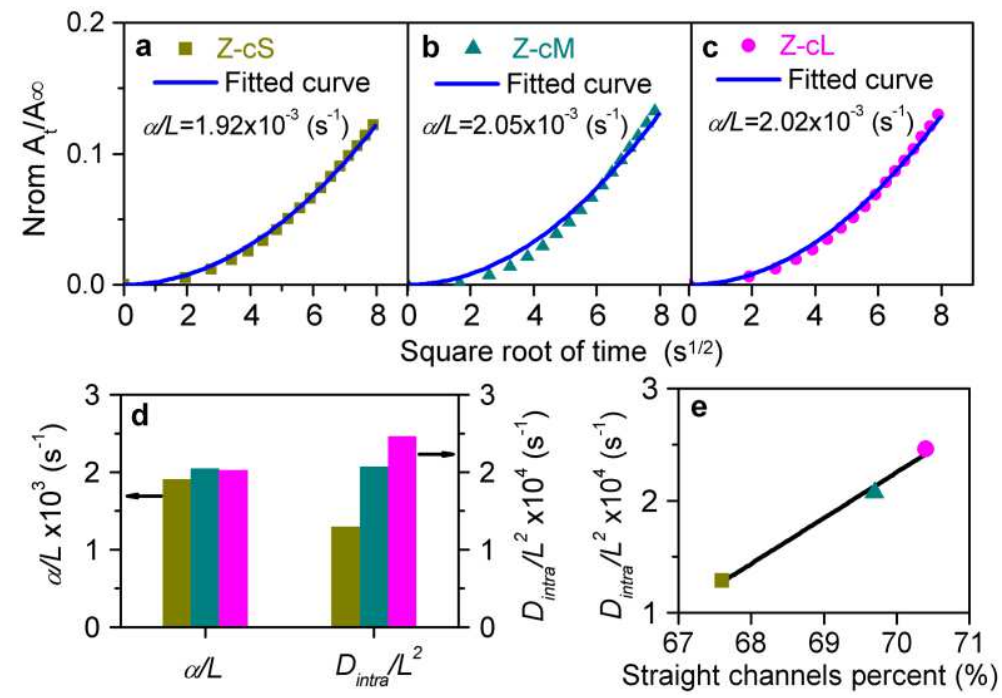

Fig. 4 Initial uptake rates of butene molecule over H-ZSM-5 zeolites: a Z-cS, b Z-cM and $\mathbf{c}$ Z-cL. d Surface permeability $(\alpha / L)$ and intracrystalline diffusion rates $\left(D_{\text {intra }} / L^{2}\right)$ of butene molecule derived by the uptake rates of H-ZSM-5 samples (as presented in Fig. 2a and Fig. 3a, b, c) following Eq. 2. e Correlation of straight channels percent computed in table 1 with the intracrystalline diffusion rates $\left(D_{\text {intra }} / L^{2}\right)$ of butene molecule.

Molecular dynamics (MD) simulations were further carried out to investigate the anisotropic diffusion of guest molecules in two channels of MFI-structured zeolite. A total of 32 propene or 24 1-butene molecules were loaded in the supercell $(2 \times 2 \times 2)$ of MFI-structured zeolite. As shown in Fig. 5, the mean square displacements (MSDs) for each molecule in $\mathrm{x}, \mathrm{y}$, and $\mathrm{z}$ directions were derived and linear relations with time are hold at the temperatures of 323,373 and $423 \mathrm{~K}$. It can be seen that the time dependence of MSDs varies obviously with the diffusion directions. It is clear that the diffusion of propene and 1-butene along the straight channel (b direction) are faster than that along the sinusoidal channels (a and $\mathrm{c}$ directions). The diffusion coefficients along $\mathrm{x}$ and $\mathrm{y}$ directions $\left(D_{\text {self-xx }}\right.$ and $\left.D_{\text {self-yy }}\right)$ were then obtained from the slopes of these curves at different temperature (Eq. 10 and Eq. 11 described in methods section), as listed in Table S4 and Table S5. Herein, the ratio of $\mathrm{D}_{\text {self-xx }} / \mathrm{D}_{\text {self-yy }}$ was employed to describe the diffusion anisotropy of guest molecules in the straight and sinusoidal channels (see Fig. 5c). It is seen that these ratios for propene and 1-butene diffusion increase monotonously with the temperature and the values are smaller than 0.6, indicating that the contribution the sinusoidal channels for the diffusion of both olefins could be enhanced with temperature. In addition, the contribution the straight channels for 
the diffusion of higher olefins increases as the ratio for propene is higher than that of 1-butene. These simulation results clearly demonstrate the diffusion anisotropy of olefins in MFI-structured zeolite, which depends on the temperature of diffusion and structure of guest molecules.
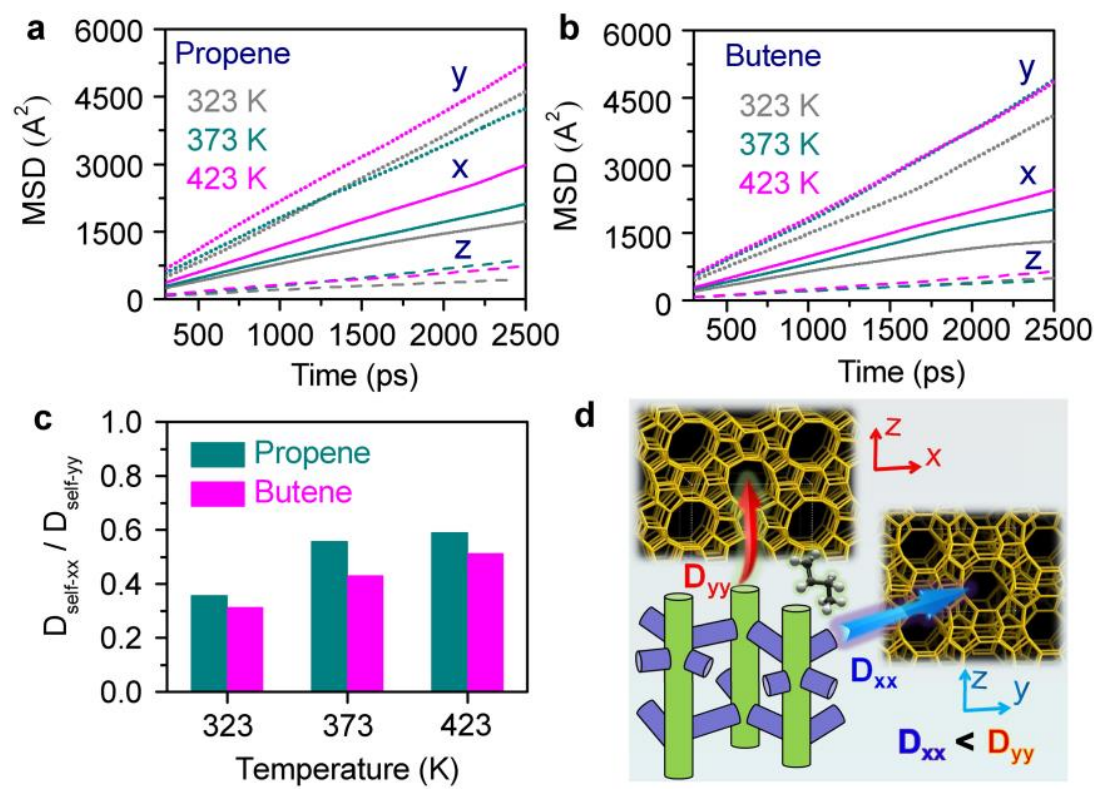

d

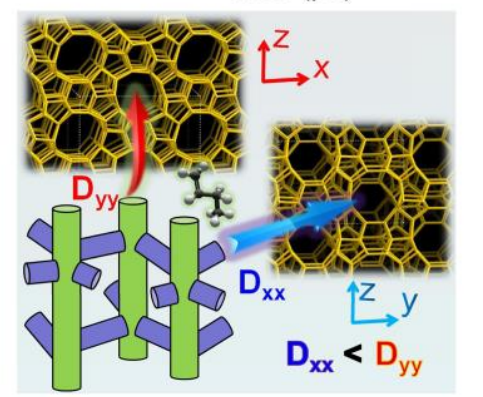

Fig. 5 Molecular dynamics simulations on the diffusion of propene and 1-butene molecules in MFI-structured zeolite. a MSDs of propene diffusion in different directions at different temperatures. b MSDs of 1-butene diffusion in different directions at different temperatures. $\mathbf{c}$ The ratio of self-diffusion coefficient in $\mathrm{x}$ - and $\mathrm{y}$ - directions. $\mathbf{d}$ Schematic view of olefin molecules diffusion along $\mathrm{x}$ - and $\mathrm{y}$ - directions of MFI type zeolites. The anisotropic components $\mathrm{MSD}_{\mathrm{xx}}$, $\mathrm{MSD}_{\mathrm{yy}}$ and $\mathrm{MSD}_{\mathrm{zz}}$ curves were calculated by three individual samples, the average MSD, $\mathrm{D}_{\text {self-xx }}$ and $\mathrm{D}_{\text {self-yy }}$ are reported in this work.

\section{Catalysis-diffusion relationship.}

The understanding of the relationship between the catalysis and the diffusion behavior is crucial to future H-ZSM-5 catalyst design for industrial catalysis. The experimental facts in Fig. 2 have pointed out an unexpected result that enlarging the particle size along c-axis over H-ZSM-5 catalyst can prolong the catalytic lifetime in OCC reaction. It was revealed that the intracrystalline diffusive rates of reactants over those selected catalysts are different due to the diverse characters of two-channels network, as shown in Fig. 3 and 4, which could lead to the differences of catalytic properties. Therefore, the catalytic properties, expressed by the deactivation rates of $\mathrm{C}_{4}=$ 
conversion and $\mathrm{C}_{2-3}=$ yield, were correlated with the intracrystalline diffusive rates of reactant molecules in internal pore channels over H-ZSM-5 catalysts, as displayed in Fig. 6a. It can be seen that the deactivation rate distinctly slowed down with the increasing of $D_{\text {intra }} / L^{2}$, indicating that the intracrystalline diffusive rate plays a pivotal role in the catalytic cracking reaction over H-ZSM-5 zeolite. For the lower olefins selectivity, as shown in Fig. S8, Z-cL with faster intracrystalline diffusivity exhibits higher $\mathrm{C}_{2-3}{ }{ }$ selectivity, while $\mathrm{Z}-\mathrm{cS}$ with stronger internal diffusion resistance shows a lower products selectivity.

In order to further investigate how intracrystalline diffusivity is related to the catalytic lifetime and products selectivity, the three H-ZSM-5 catalysts were comparatively analyzed using TGA and Ar adsorption after $50 \mathrm{~h}$ of reaction (Fig. S9 and Table S6). Particularly, the locations and the amounts of coke formation calculated using the method reported by Ryoo et al. ${ }^{39}$, were studied as a function of the intracrystalline diffusivity. As the result in Fig. 6b shows, although the total coke contents are similar among these three catalysts, it is of interest that the ratios of external and internal coke species are quite different. It was suggested that the coke species deposited inside the micropores is believed to induce the quick deactivation in catalysis ${ }^{39,40}$. The acceleration of $D_{\text {intra }} / L^{2}$ increased the fraction of external coke species rather than that of internal cokes inside the micropores, and decreased the microporous blockage degree, hence contributing to prolonging the catalytic lifetime. Thus, a faster intracrystalline diffusive rate would promote the reactants and products move outward the crystals during OCC reaction and delay further transformation of light olefins to heavy cokes in the micropores, which increases the resistance to deactivation and lower olefins selectivity. 


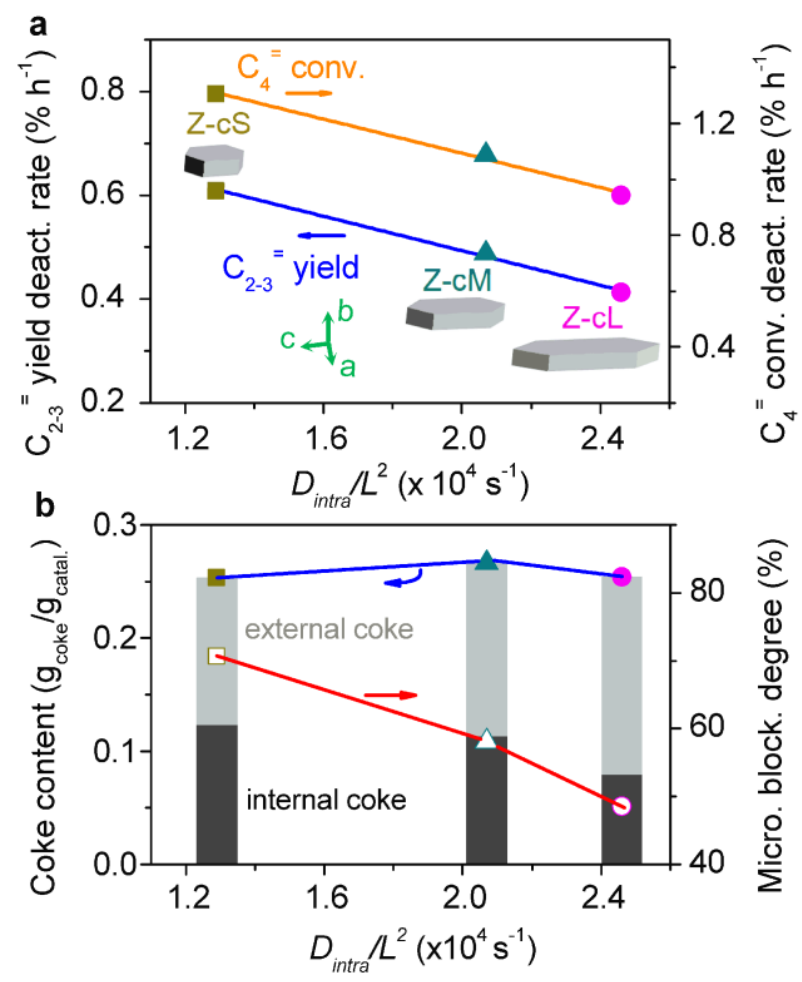

Fig. 6 a Correlation of $\mathrm{C}_{4}{ }^{=}$conversions and $\mathrm{C}_{2-3}{ }^{=}$yield deactivation rates with the intracrystalline diffusive rates of butene molecules in H-ZSM-5 crystals. The deactivation rates were calculated on the basis of the catalytic performances displayed in Fig. 2a and b. The data between $20 \mathrm{~h}$ and $50 \mathrm{~h}$ were chosen due to the actual deactivation time. $\mathbf{b}$ The coke depositions and the micropores blockage degrees after $50 \mathrm{~h}$ of OCC reactions.

It has been demonstrated that the intracrystalline diffusion in H-ZSM-5 crystal, which significantly impacts the catalytic performance, is closely related to the percent of straight channels due to their molecules diffusive propensity. Therefore, we consider that the anisotropic diffusion behavior in two-channels network of H-ZSM-5 crystal is the essential mechanism for morphology effect in olefin catalytic cracking. The controlling of the percent of straight channels by prolonging the c-axis morphology in H-ZSM-5 could be applied to modulate catalyst lifetime and products yield in olefin catalytic cracking.

\section{Discussion}

In this work, A series of H-ZSM-5 zeolites with controllable nano-sheet morphology were precisely designed: the acidities, the textures, the sizes along a- and b-axis are comparative, but the lengths of c-axis are variable, suggesting that the compositions and the diffusive path lengths 
over these zeolites are constant, but the percents of pore channels are diverse. Then, the catalytic performances of these H-ZSM-5 zeolites in olefin catalytic cracking were investigated. It was pointed out that enlarging the particle size along c-axis in H-ZSM-5 catalyst will unexpectedly improve the catalytic activity and stability, which is distinct from the conventional rules of mass transfer behaviors in zeolites. Furthermore, based on the time-resolved in-situ FTIR spectroscopy, It was found that the apparent diffusive rates of the guest molecules were significantly boosted with the increase of exposed [010] plane degrees over H-ZSM-5 zeolites. According to the analysis of the dual resistance model (DRM), it was demonstrated that the morphology effect of diffusive properties was essentially related to the anisotropic diffusion in different channels of H-ZSM-5 zeolite. The intracrystalline diffusive rate in straight channels is faster than that in sinusoidal channels for olefins molecules, which was further confirmed by molecular dynamic simulations (MD). Therefore, the diffusion anisotropy in different channels was proposed as the descriptor for the morphology effect in olefin catalytic cracking. The controlling of the intracrystalline diffusive rate via changing the proportion of pore channels could modulate the catalytic activity and stability due to the differences in the location of the coke species in OCC reaction. In short, this work not only provides a clear diffusion anisotroy descriptor to reveal the morphology effect, but also offers a deep insight into design of highly effective zeolite catalysts for OCC process.

\section{Methods}

Synthesis of H-ZSM-5 catalysts. The zeolite materials were prepared by hydrothermal synthesis according to previously reported conventional method ${ }^{11}$. The synthesis of H-ZSM-5 zeolites was carried out by dissolving $\mathrm{Al}_{2}\left(\mathrm{SO}_{4}\right)_{3} \cdot 18 \mathrm{H}_{2} \mathrm{O}$ in the solution of sodium hydroxide, followed by the addition of silica solution under stirring. Zeolite synthesis using additives was performed at an original gel composition at $6 \mathrm{TPA}^{+}: 20 \mathrm{SiO}_{2}: 2.5 \mathrm{Na}_{2} \mathrm{O}: 0.025$ $\mathrm{Al}_{2} \mathrm{O}_{3}:(0-1)$ pyrocatechol:80 EtOH:800 $\mathrm{H}_{2} \mathrm{O}$, under basic solution with the help of Free Slate High-throughput equipment. The precursor was aged for $24 \mathrm{~h}$ and heated to $160{ }^{\circ} \mathrm{C}$ under $150 \mathrm{rpm}$ stirring for 2 days. After washed and filtered for several times, the products were collected, centrifuged, filtered, and dried in air at the oven. Finally, the samples were calcinated at $550^{\circ} \mathrm{C}$ for $5 \mathrm{~h}$ to remove the organic template.

Characterizations of H-ZSM-5 catalysts. X-ray powder diffraction (XRD) patterns were recorded on a Bruker D8 Advance diffractometer. The $\mathrm{Cu} K \alpha$ radiation $(\lambda=5.1540589 \mathrm{~nm})$ generated at $40 \mathrm{kV}$ and $40 \mathrm{~mA}$ was used as 
the X-ray source with the scanning rate $1^{\circ} \mathrm{min}^{-1}$ over a $2 \theta$ range of $5-50^{\circ}$. Ar physisorption measurements were performed on a Micromeritics TriStar3000 Surface Area Analyzer at 75 K. Prior to the measurements, the sample was degassed at $350{ }^{\circ} \mathrm{C}$ until a stable vacuum of about $0.67 \mathrm{~Pa}$ was reached. The acidity of the H-ZSM-5 samples (Brönsted acid sites) were studied by pyridine-adsorption in an in situ IR cell with $\mathrm{CaF}_{2}$ windows. Self-supported sample discs with weight of $15 \mathrm{mg}$ and diameter $13 \mathrm{~mm}$ were pretreated at $400{ }^{\circ} \mathrm{C}$ for $4 \mathrm{~h}$ under ultrahigh vacuum system. Subsequently, the sample was cooled down to $200^{\circ} \mathrm{C}$, pyridine steams were admitted to the IR cell for 30 min. Then physical adsorption pyridine was eliminated by evacuation of the zeolite sample for $30 \mathrm{~min}$, and the spectra were recorded on a Nicolet 6700 instrument equipped with an MCT detector at a resolution of $4 \mathrm{~cm}^{-1}$. FE-SEM (Field Emission Scanning Electron Microscopy) analysis was performed on a Hitachi S4800 electron microscope. The sample was dispersed ultrasonically in ethanol for $10 \mathrm{~min}$, and then was dropped onto a silicon pellet, followed by drying for $60 \mathrm{~min}$. More than 100 particles were used to evaluate the mean length of a, b and c axis from SEM images. TEM images were performed on a Tecnai 20 STWIN electron microscope at $200 \mathrm{kV}$. The aberration-corrected scanning transmission electron microscopy (STEM) images were obtained on a FEI Titan Cubed Themis G2 $300 \mathrm{kV}$ with an accelerating voltage of $300 \mathrm{kV}$.

Coke analysis. The H-ZSM-5 zeolite after $50 \mathrm{~h}$ of reaction was taken for thermogravimetric analysis (TGA SDT Q600 V20.9 Build 20). The temperature was increased to $1123 \mathrm{~K}$ under flowing air $\left(100 \mathrm{~mL} \mathrm{~min}^{-1}\right)$ at a constant ramping rate of $10 \mathrm{~K} \mathrm{~min}^{-1}$. The weight loss between 673 and $1073 \mathrm{~K}$ was regarded as the total coke content. Based on the assumption that the coke density was $1.22 \mathrm{~g} \mathrm{~cm}^{-3}$, which is similar to that of coal ${ }^{39,41}$. On one hand, the content of internal coke species in the micropores was calculated by the micropore volume decrease, as compared with the fresh zeolite micropore volume. On the other hand, The content of external coke species outside the external surface was determined from the total coke content subtracting the internal coke content. The micropores blockage degree was obtained by the ratio of micropore volume decrease to the fresh H-ZSM-5 micropore volume.

Calculations of the surface areas of various exposure facets. For the coffin-like H-ZSM-5 crystal, the surface areas of [010], [100] and [101] crystal plane were calculated by the following formula:

$$
\begin{aligned}
& S_{[100]}=2 \times L_{\mathrm{b}} \times\left(L_{c}-2 \times \frac{L_{\mathrm{a}}}{2 \times \tan \left(118^{\circ} / 2\right)}\right) \\
& S_{[010]}=2 \times L_{\mathrm{a}} \times\left(L_{\mathrm{c}}-\frac{L_{\mathrm{a}}}{2 \times \tan \left(118^{\circ} / 2\right)}\right) \\
& S_{[101]}=4 \times L_{\mathrm{b}} \times \frac{L_{\mathrm{a}}}{2 \times \sin \left(118^{\circ} / 2\right)}
\end{aligned}
$$

Where $\mathrm{L}_{a}, \mathrm{~L}_{b}, \mathrm{~L}_{c}$ represents the length of $\mathrm{a}, \mathrm{b}$ and $\mathrm{c}$-axis in table $1.118^{\circ}$ represents the angle between two intersecting [101] crystal plane in coffin-like H-ZSM-5 crystal. 
Catalytic cracking process. The catalytic cracking of butene reactions were performed in a stainless catalytic reactor. The catalyst $(0.30 \mathrm{~g})$ with grain sizes of 20-40 mesh was loaded in the reactor and was pretreated in high-purity $\mathrm{N}_{2}$ flow at $823 \mathrm{~K}$ for $2 \mathrm{~h}$. Then, pure $\mathrm{C}_{4}$ olefin with WHSV of $30^{-1} \mathrm{~h}$ was introduced into the reactor with a laboratory-scale piston pump for $\mathrm{C}_{4}$-olefin input. The pressure of reactant was typically regulated at 1.6 bar. Products were analyzed by an online gas chromatography (Agilent 7860). The calculation of $\mathrm{C}_{4}=$ conversion was based on the moles of $\mathrm{C}_{4}{ }^{=}$at inlet and outlet gases, the product selectivity was calculated on a molar carbon basis.

Measurements of diffusivity. The diffusion properties of guest molecules over as-synthesized H-ZSM-5 samples were evaluated by a home made time-resolved in situ FT-IR spectroscopy. Karge and Niessen firstly exploited this technique to study the diffusion behaviors of guest molecules over zeolites at $1991 \mathrm{~s}^{42}$, the counter- or co-diffusion behaviors of hydrocarbons in zeolietes could be determined under a rigorous conditions of high temperatures and pressures $^{42-45}$. The IR spectra were collected on a Nicolet 6700 instrument equipped with an MCT detector at a resolution of $4 \mathrm{~cm}^{-1}$. Firstly, the H-ZSM-5 sample was pressed into a self-supporting wafer with a diameter of 13 $\mathrm{mm}$ at the pressed pressure of $30 \mathrm{MPa}$ and placed inside a gold sample holder surrounded by a heating wire. The sample holder was fixed in the in situ IR cell connected to an air control system. Before measurement, H-ZSM-5 sample was pretreated with a $100 \mathrm{~mL} \mathrm{~min}^{-1}$ pure $\mathrm{N}_{2}$ flow at $673 \mathrm{~K}$ for $2 \mathrm{~h}$. Subsequently, the sample was cooled down to the test temperature with a $800 \mathrm{~mL} \mathrm{~min}^{-1} \mathrm{~N}_{2}$ flow rate, after stabilizing at the targeted temperature, the background spectrum was obtained as a reference in pure $\mathrm{N}_{2}$ flow. For a typical measurement, the guest molecules $\left(\mathrm{C}_{4}=\right.$ or $\left.\mathrm{C}_{3}{ }^{=}\right)$mixing with inert $\mathrm{N}_{2}\left(0.1 \mathrm{vol} \%\right.$ guest molecule $\left.+99.9 \mathrm{vol} \% \mathrm{~N}_{2}\right)$ with a partial pressure of 1 mbar was introduced into the IR cell with a $800 \mathrm{~mL} \mathrm{~min}^{-1}$ flow, and the IR spectra were collected at $0.96 \mathrm{~s}$ intervals. The normalized areas of the IR bands of butene and propene molecules at $2750-3150 \mathrm{~cm}^{-1}$ were used to quantify the relative concentrations of adsorbate in the zeolites. Finally, the original and normalized uptake curves were obtained, and the diffusion rates were calculated using the following formula for sheet like zeolite ${ }^{46,47}$ :

$$
\frac{m_{t}}{m_{(t \rightarrow \infty)}}=1-\frac{8}{\pi^{2}} \sum_{n=0}^{\infty} \frac{1}{(2 n+1)^{2}} \exp \left(-(2 n+1)^{2} \frac{\pi^{2} D_{e f f}}{L^{2}} t\right)
$$

Where $\mathrm{m}_{\mathrm{t}}$ is the amount adsorbed at time $\mathrm{t}$ (in $\mathrm{s}$ ), $\mathrm{m}_{\infty}$ is the amount adsorbed at equilibrium coverage, $D_{\text {eff }}$ represents the apparent diffusion coefficient, and $L$ represents the diffusion length of H-ZSM-5 crystal. In this work, the diffusion rate, $D_{e f f} L^{2}$ was chosen for evaluated the diffusion property of H-ZSM-5 sample.

Computational methods. molecular dynamics (MD) simulations by Forcite module in materials studio 8.0 with COMPASS-II force field were performed to investigate the diffusion properties in two-channels network over MFI type zeolite. Guest molecules loadings in pure silica zeolite were set at 32 and 24 molecules per supercell $(2 \times 2 \times 2)$ for propene and butene molecule respectively, ensuring that the number of heavy atoms in each simulation was consistent. The initial structural model for each MD simulation was obtained by the Packing technology in the Amorphous Cell module. All MD simulations were performed in NVT ensemble and the temperatures were maintained by the Nose thermostat. The long-range interaction was calculated by Ewald summation method with a 
cutoff radius $15.5 \AA$. The velocity Verlet integrator is used with a time step of $1 \mathrm{fs}$. Snapshots of the positions were recorded at every $0.5 \mathrm{ps}$. The simulations studies were performed at various temperatures of 323 , 373, and $423 \mathrm{~K}$, each for $5 \mathrm{~ns}$, following an equilibration of $0.3 \mathrm{~ns}$. According to the dynamic trajectory of each system, the mean square displacement (MSD) of an adsorbate molecule during a time interval $\tau$ was calculated by the following equation:

$\operatorname{MSD}(\tau)=\frac{1}{N_{m}} \sum_{\mathrm{i}}^{N_{\mathrm{m}}} \frac{1}{N_{i}} \sum_{t_{0}}^{N_{i}}\left|r_{i}\left(t_{0}+\tau\right)-r_{i}\left(t_{0}\right)\right|^{2}$

Where $\mathrm{N}_{\mathrm{m}}$ corresponds to the number of olefin molecules considered in the calculation of the MSD. Therefore, the self-diffusion coefficient $D_{\text {self }}$ was obtained by fitting the MSD plots respecting to the time range $0.3 \sim 2.5 \mathrm{~ns}$.

$\operatorname{MSD}(\tau)=6 D_{\text {self }} \tau+b$

Where $b$ is the offset at time zero. In addition to the isotropic average, the anisotropic components of MSD (i.e., xx, yy, zz,) were also produced with the following equation:

$M S D=M S D_{x x}+M S D_{y y}+M S D_{z z}$

The self-diffusion coefficient $D_{\text {self-xx }}$ and $D_{\text {self-yy }}$ in the x- (sinusoidal channels of MFI type zeolite) and y- (straight channels of MFI type zeolite) directions could be respectively obtained by fitting the separate plots $\mathrm{MSD}_{\mathrm{xx}}$ and $\mathrm{MSD}_{\mathrm{yy}}$ :

$M S D_{\mathrm{xx}}(\tau)=2 D_{\text {self }-x x} \tau+b$

$M S D_{\mathrm{yy}}(\tau)=2 D_{\text {self }-y y} \tau+b$

\section{Data availability}

The data supporting the findings of this study are available from the corresponding authors on reasonable request.

\section{References}

1. Corma, A. From microporous to mesoporous molecular sieve materials and their use in catalysis. Chem. Rev. 97, 2373-2419 (1997).

2. Yarulina, I. et al. Structure-performance descriptors and the role of Lewis acidity in the methanol-to-propylene process. Nature Chem. 10, 804-812 (2018). 
3. Kokotailo, G. T. Lawton, S. L. Olson, D. H. \& Meier, W. M. Structure of synthetic zeolite ZSM-5. Nature 272, 437-438 (1978).

4. Olson, D. H. Kokotailo, G. T. Lawton, S. L. \& Meier, W. M. Crystal structure and structure-related properties of ZSM-5, J. Phys. Chem. 85, 2238-2243 (1981).

5. Karwacki, L. et al. Morphology-dependent zeolite intergrowth structures leading to distinct internal and outer-surface molecular diffusion barriers. Nat. Mater. 8, 959-965 (2009).

6. Shi, J. et al. Recent advances of pore system construction in zeolite-catalyzed chemical industry processes. Chem. Soc. Rev. 44, 8877-8903 (2015).

7. del Campo, P. et al. Propene production by butene cracking. descriptors for zeolite catalysts. ACS Catal. 10, 11878-11891 (2020).

8. Choi, M. et al. Stable single-unit-cell nanosheets of zeolite MFI as active and long-lived catalysts. Nature, 461, 246-250 (2009).

9. Wang, N. et al. Modulation of b-axis thickness within MFI zeolite: Correlation with variation of product diffusion and coke distribution in the methanol-tohydrocarbons conversion. Appl. Catal. B 243, 721-733 (2019).

10. Khare, R. Millar, D. \& Bhan, A. A mechanistic basis for the effects of crystallite size on light olefin selectivity in methanol-to-hydrocarbons conversion on MFI. J. Catal. 321, 23-31 (2015).

11. Shi, J. et al. Morphology control of ZSM-5 zeolites and their application in Cracking reaction of $\mathrm{C}_{4}$ olefin. Inorg. Chem. Front. 5, 2734-2738 (2018).

12. Wang, C. et al. Maximizing sinusoidal channels of HZSM-5 for high shape-selectivity to p-xylene. Nature Comm. 10, 4348 (2019).

13. Fu, D. et al. Disentangling reaction processes of zeolites within single oriented channels. Angew. Chem. Int. Ed. 59, 15502-15506 (2020).

14. Wang, N. et al. Crystal-plane effects of MFI zeolite in catalytic conversion of methanol to hydrocarbons. J. Catal. 360, 89-96 (2018).

15. Kärger, J. Random walk through two-channel networks: a simple means to correlate the coefficients of anisotropic diffusion in ZSM-5 type zeolites, J. Phys. Chem. 95, 5558-5560 (1991). 
16. June, R. L. Bell, A. T. \& Theodorou, D. N. Molecular dynamics study of methane and xenon in silicalite. J. Phys. Chem. 94, 8232-8240 (1990).

17. Hong, U. Kärger, J. Pfeifer, H. Müller, U. \& Unger, K. K. Observing diffusion anisotropy in zeolites by pulsed field gradient NMR. Z. Phys. Chem. 173, 225-234 (1991)

18. June, R. L. Bell, A. T. \& Theodorou, D. N. Molecular dynamics studies of butane and hexane in siiicalite. J. Phys. Chem. 96, 1051-1060 (1992).

19. Zeng, S. et al. Differentiating diffusivity in different channels of ZSM-5 zeolite by pulsed field gradient (PFG) NMR. ChemCatChem 12, 463-468 (2020).

20. Blay, V., Miguel, P. J., \& Corma, A. Theta-1 zeolite catalyst for increasing the yield of propene when cracking olefins and its potential integration with an olefin metathesis unit. Catal. Sci. Technol. 7, 5847-5859 (2017).

21. Chen, J. Q., Bozzano, A., Glover, B., Fuglerud, T., \& Kvisle, S. Recent advancements in ethene and propene production using the UOP/Hydro MTO process. Catal. Today 106, 103-107 (2005).

22. Blay, V. Epelde, E. Miravalles, R. \& Perea, L. A. Converting olefins to propene: Ethene to propene and olefin cracking. Catal. Rev. 60, 278-335 (2018).

23. Lukyanov, D. B. Shtral, V. I. \& Khadzhiev, S. N. A kinetic model for the hexane cracking reaction over H-ZSM-5. J. Catal. 146, 87-92 (1994).

24. Mazar, M. N. Al-Hashimi, S. Cococcioni, M. \& Bhan, A. $\beta$-scission of olefins on acidic zeolites: a periodic PBE-D study in H-ZSM-5. J. Phys. Chem. C 117, 23609-23620 (2013).

25. Chen, C. J. Rangarajan, S. Hill, I. M. \& Bhan, A. Kinetics and thermochemistry of $\mathrm{C}_{4}-\mathrm{C}_{6}$ olefin cracking on H-ZSM-5. ACS Catal. 4, 2319-2327 (2014).

26. Zhao, G. L. et al. Effect of phosphorus on HZSM-5 catalyst for C4-olefin cracking reactions to produce propylene. J. Catal. 248, 29-37 (2007).

27. Zhou, J. et al. Full-crystalline hierarchical monolithic ZSM-5 zeolites as superiorly active and long-lived practical catalysts in methanol-to-hydrocarbons reaction. J. Catal. 340, 166-176 (2016).

28. Yang, J. H. et al. Enhanced aromatic selectivity by the sheet-like ZSM-5 in syngas conversion. J. Energy Chem. 35, 44-48 (2019). 
29. Zhao, G. L. et al. Synthesis of ZSM-48 zeolites and their catalytic performance in C4-olefin cracking reactions. Appl. Catal. A Gen. 299, 167-174 (2006).

30. Hibbe, F. et al. The nature of surface barriers on nanoporous solids explored by microimaging of transient guest distributions. J. Am. Chem. Soc. 133, 2804-2807 (2011).

31. Heinke, L. \& Kärger, J. Correlating surface permeability with intracrystalline diffusivity in nanoporous solids. Phys. Rev. Lett. 106, 074501 (2011).

32. Qi, X., Vattipalli, V., Dauenhauer, P. J. \& Fan, W. Silica nanoparticle mass transfer fins for MFI composite materials. Chem. Mater. 30, 2353-2361 (2018).

33. Krishna, R. Diffusion in porous crystalline materials. Chem. Soc. Rev. 41, 3099-3118 (2012).

34. Beerdsen, E., Dubbeldam, D. \& Smit, B. Understanding diffusion in nanoporous materials. Phys. Rev. Lett. 96, 044501 (2006).

35. Gao, M. et al. Direct quantification of surface barriers for mass transfer in nanoporous crystalline materials. Commun. Chem. 2, (2019).

36. Peng, S. et al. Control of surface barriers in mass transfer to modulate methanol-to-olefins reaction over SAPO-34 zeolites. Angew. Chem. Int. Ed. DOI:10.1002/anie.202009230 (2020).

37. Zhang, L. et al. Direct assessment of molecular transport in mordenite: dominance of surface resistances. Chem. Commun. 42, 6424-6426 (2009).

38. Tzoulaki, D. et al. Assessing Surface Permeabilities from Transient Guest Profiles in Nanoporous Host Materials. Angew. Chem. Int. Ed. 48, 3525 (2009).

39. Kim, J., Choi, M. \& Ryoo, R. Effect of mesoporosity against the deactivation of MFI zeolite catalyst during the methanol-to-hydrocarbon conversion process. J. Catal. 269, 219-228, (2010).

40. Shen, X. et al. Impact of hierarchical pore structure on the catalytic performances of MFI zeolites modified by $\mathrm{ZnO}$ for the conversion of methanol to aromatics. Catal. Sci. Technol. 7, 3598-3612 (2017).

41. Bibby, D. M. et al. Coke formation in zeolite ZSM-5. J. Catal. 97, 493 (1986).

42. Karge, H. G. \& Niessen, W. A new method for the study of diffusion and counter-diffusion in zeolites. Catal. Today 8, 451-485 (1991). 
43. Süer, M. G. et al. In-situ CIR-FTIR study of the diffusion supercritical hydrocarbons in zeolite L. AIChE J. 43, 1717-1726 (1997).

44. Roque-Malherbe, R. Wendelbo, R. Mifsud, A. \& Corma, A. Diffusion of aromatic hydrocarbons in H-ZSM-5, H-Beta, and H-MCM-22 zeolites. J. Phys. Chem. 99, 14064-14071 (1995).

45. Karge, H. G. Infrared spectroscopic investigation of diffusion, co-diffusion and counter-diffusion of hydrocarbon molecules in zeolites. C. R. Chimie 8, 303-319 (2005).

46. Karger, J. \& Ruthven, D. M. Diffusion in nanoporous materials: fundamental principles, insights and challenges. New J. Chem. 40, 4027 (2016).

47. Chmelik, C. \& Karger, J. In situ study on molecular diffusion phenomena in nanoporous catalytic solids. Chem. Soc. Rev. 39, 4864-4884 (2010).

\section{Acknowledgements}

We greatly acknowledge the financial support from the Chinese Postdoctoral Science Foundation (2020M681445), the Shanghai Rising-Star Program (18QB1404500). We would like to greatly thank Dr. Hao Tian, Dr. Xiejun Huang and Dr. Jinshu Tian reviewed the manuscript and offering very helpful suggestions.

\section{Author contributions}

X. Liu performed most of the experiments and analyzed the experimental data. X. Liu also co-wrote the paper. J. Teng and Y. Wang analyzed the data and co-wrote the paper. J. Shi synthesized most of the zeolite catalysts. G. Yang and C. Wang performed MD simulations. J. Zhou analyzed the results, performed and reviewed the paper. Z. Xie designed the study, analyzed the experimental results and co-wrote the paper. All authors discussed the results and analyzed the manuscript. 


\section{Figures}

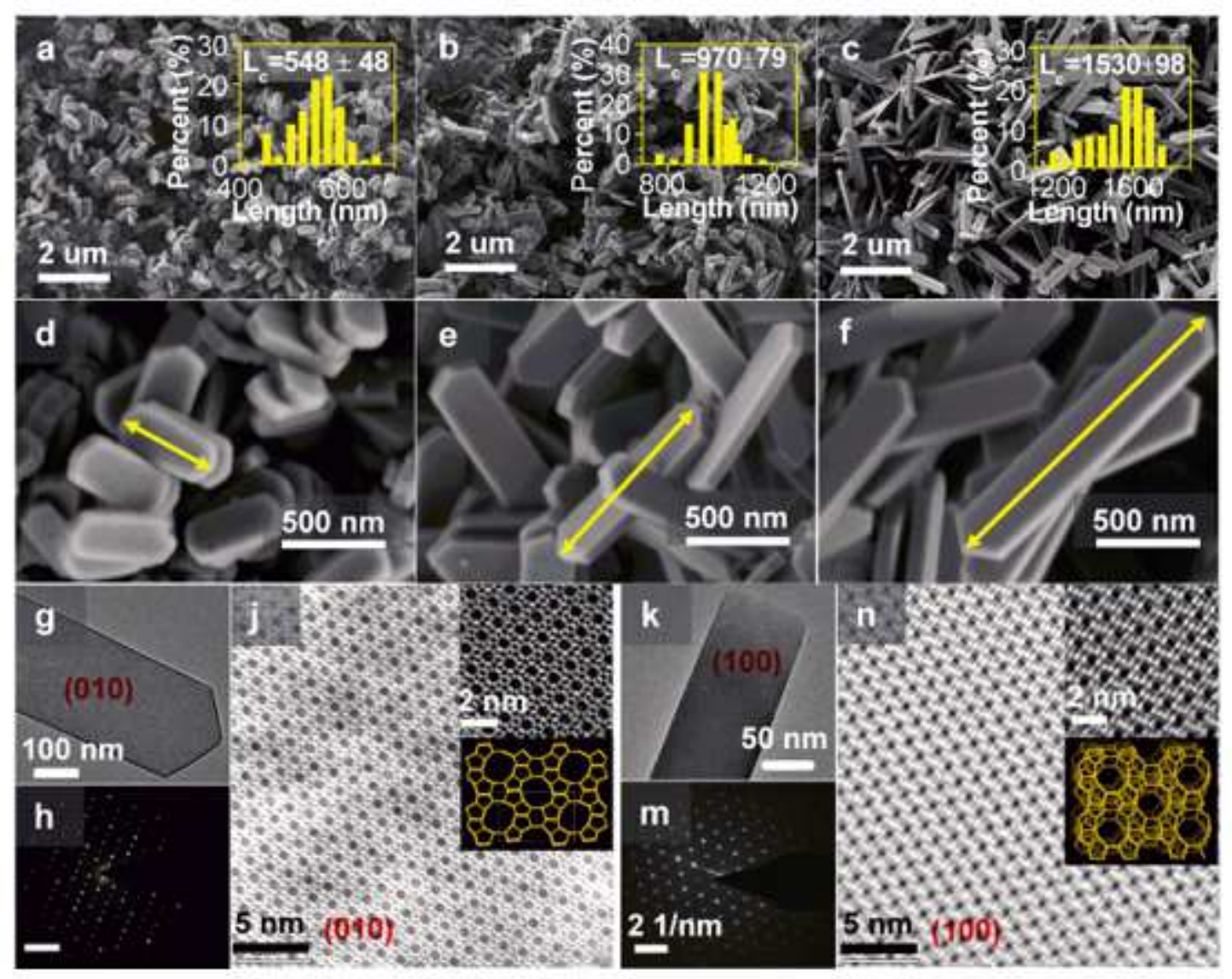

\section{Figure 1}

The morphology of the as-synthesized H-ZSM- 5 samples. SEM results of H-ZSM- 5 zeolites with similar thicknesses of $a$ and $b$ axis but different lengths of $c$ axis: $a, d$ Z-cS; b, e Z-cM; $c, f$ Z-cL. $g$ TEM image of $Z-c L$ sample with exposed facets of [010] plane. $h$ The corresponding SAED patterns of [010] plane. $j$ Aberration-corrected STEM image of Z-cL sample with exposed facets of [010] plane, with the inset on the top right displaying the STEM image with high resolution and the framework structure of $\mathrm{H}-\mathrm{ZSM}-5$ projected along [010] plane. $k, m, n$ TEM image, the corresponding SAED patterns and the aberrationcorrected STEM image of Z-cL sample with exposed facets of [100] plane. 

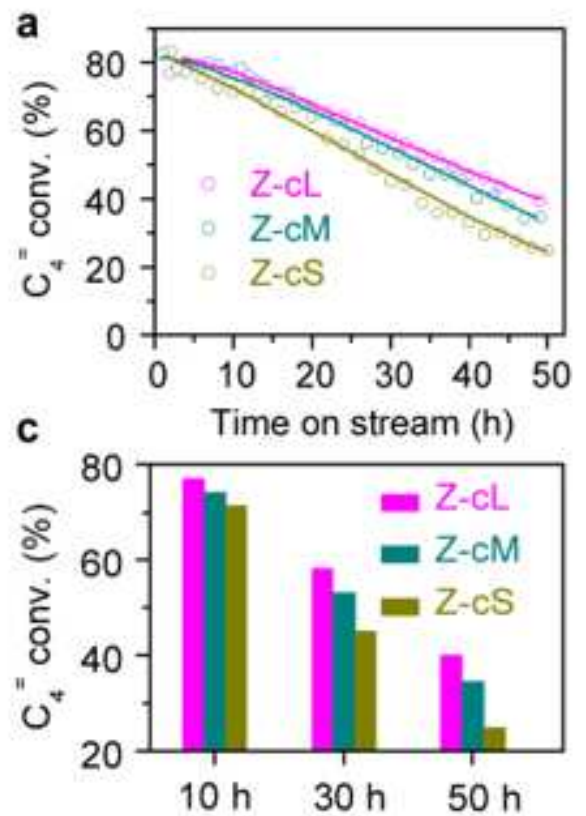

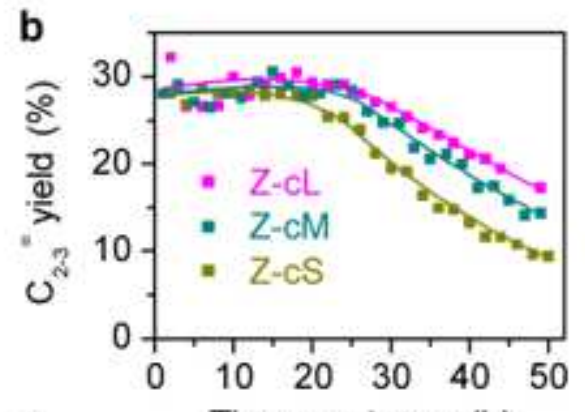

d Time on stream (h)

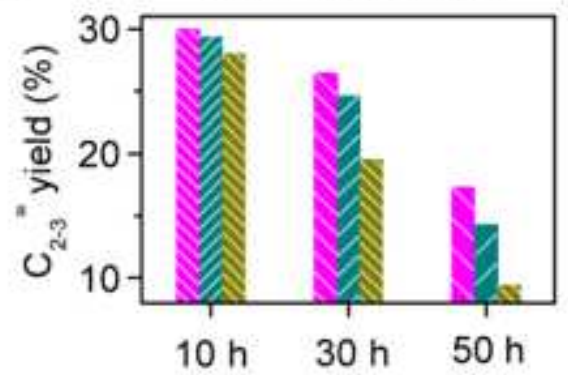

\section{Figure 2}

Catalytic behaviors for $\mathrm{C} 4$ olefins catalytic cracking reactions over Z-cS, Z-cM and Z-cL catalysts. a C4= conversions. $\mathrm{b}$ Yields of propene and ethene. $\mathrm{c}, \mathrm{d}$ detailed $\mathrm{C} 4=$ conversions and $\mathrm{C} 2-3=$ yields at different reaction times. Reaction conditions: $\mathrm{M}$ (catalyst) $=0.3 \mathrm{~g} ; \mathrm{P}=1.6 \mathrm{Bar} ; \mathrm{T}=823 \mathrm{~K} ; \mathrm{F}=15 \mathrm{~mL} \mathrm{h-1;}$ Time on stream, $50 \mathrm{~h}$.
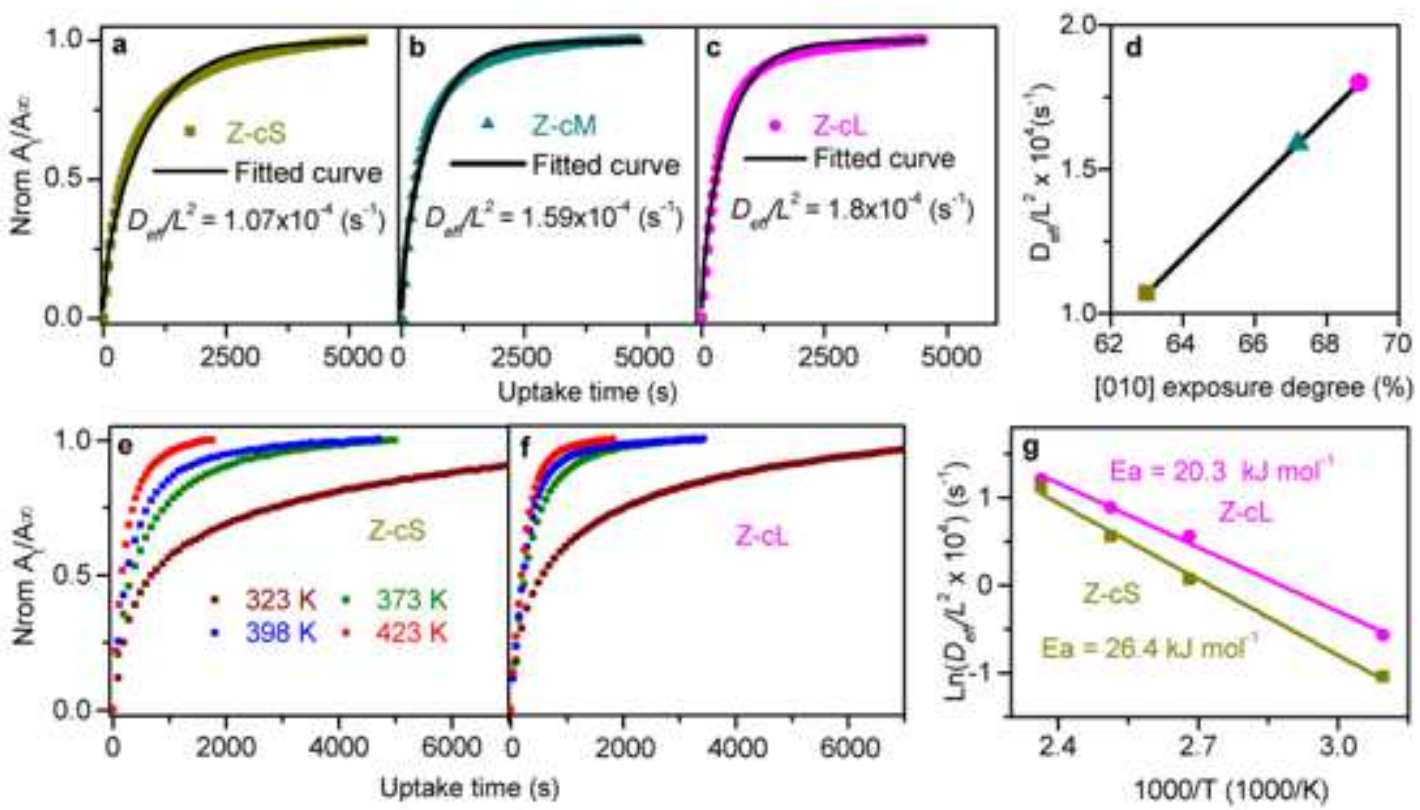

Figure 3

Diffusion behaviors of $\mathrm{C} 4=$ in $\mathrm{H}-\mathrm{ZSM}-5$ zeolites. Uptake curves of $\mathrm{C} 4=$ over $\mathrm{H}-\mathrm{ZSM}-5$ samples with different length of c axis: a Z-cS, b Z-cM, c Z-cL. d Correlation of the [010] exposure degrees of H-ZSM-5 
zeolites with the diffusion rates fitted by Eq.6. e, $\mathrm{f}$ Uptake curves of $\mathrm{C} 4=$ over Z-cS and Z-cL with different temperature. g Arrhenius plot of diffusion rates over Z-cS and Z-cL. The detailed conditions of diffusive measurements are displayed in methods section.
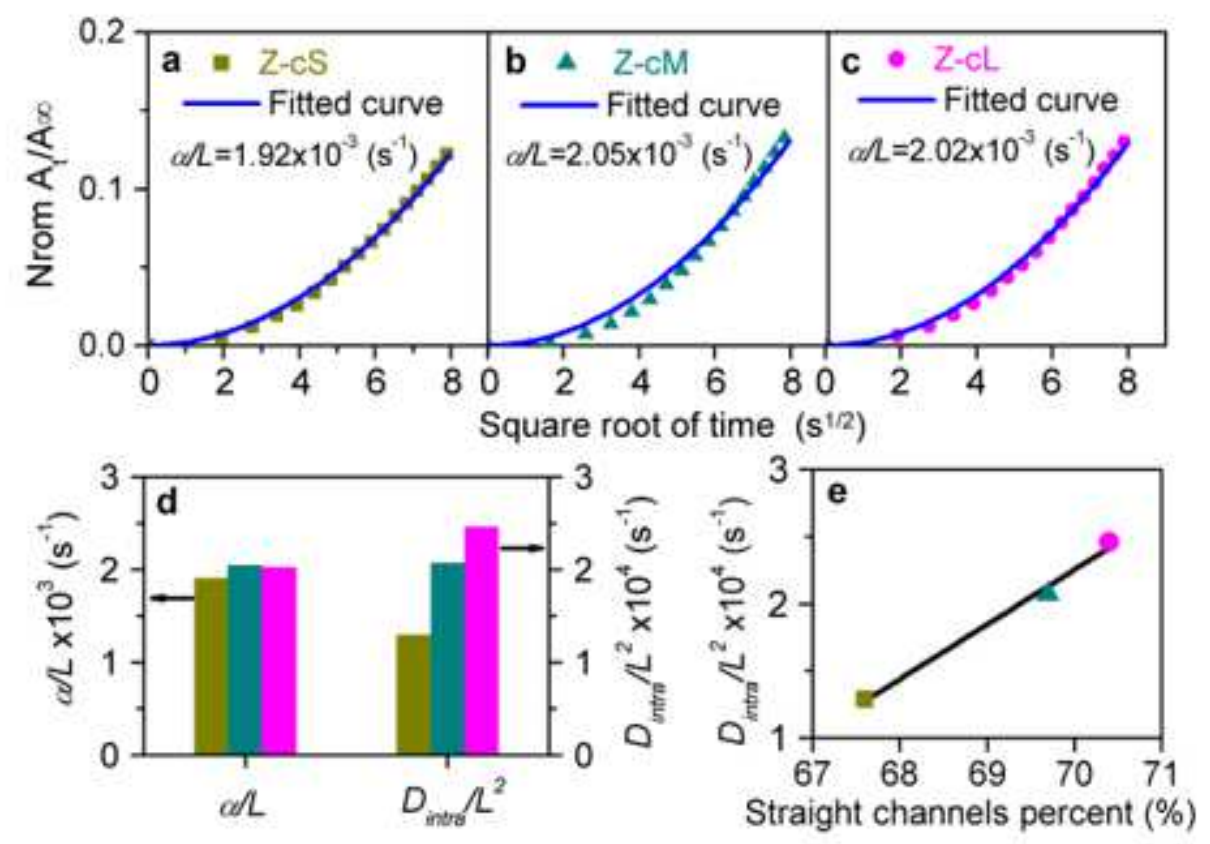

\section{Figure 4}

Initial uptake rates of butene molecule over H-ZSM-5 zeolites: a Z-cS, b Z-cM and c Z-cL. d Surface permeability $(a / L)$ and intracrystalline diffusion rates (Dintra/L2) of butene molecule derived by the uptake rates of H-ZSM-5 samples (as presented in Fig. 2a and Fig. 3a, b, c) following Eq. 2. e Correlation of straight channels percent computed in table 1 with the intracrystalline diffusion rates (Dintra/L2) of butene molecule. 

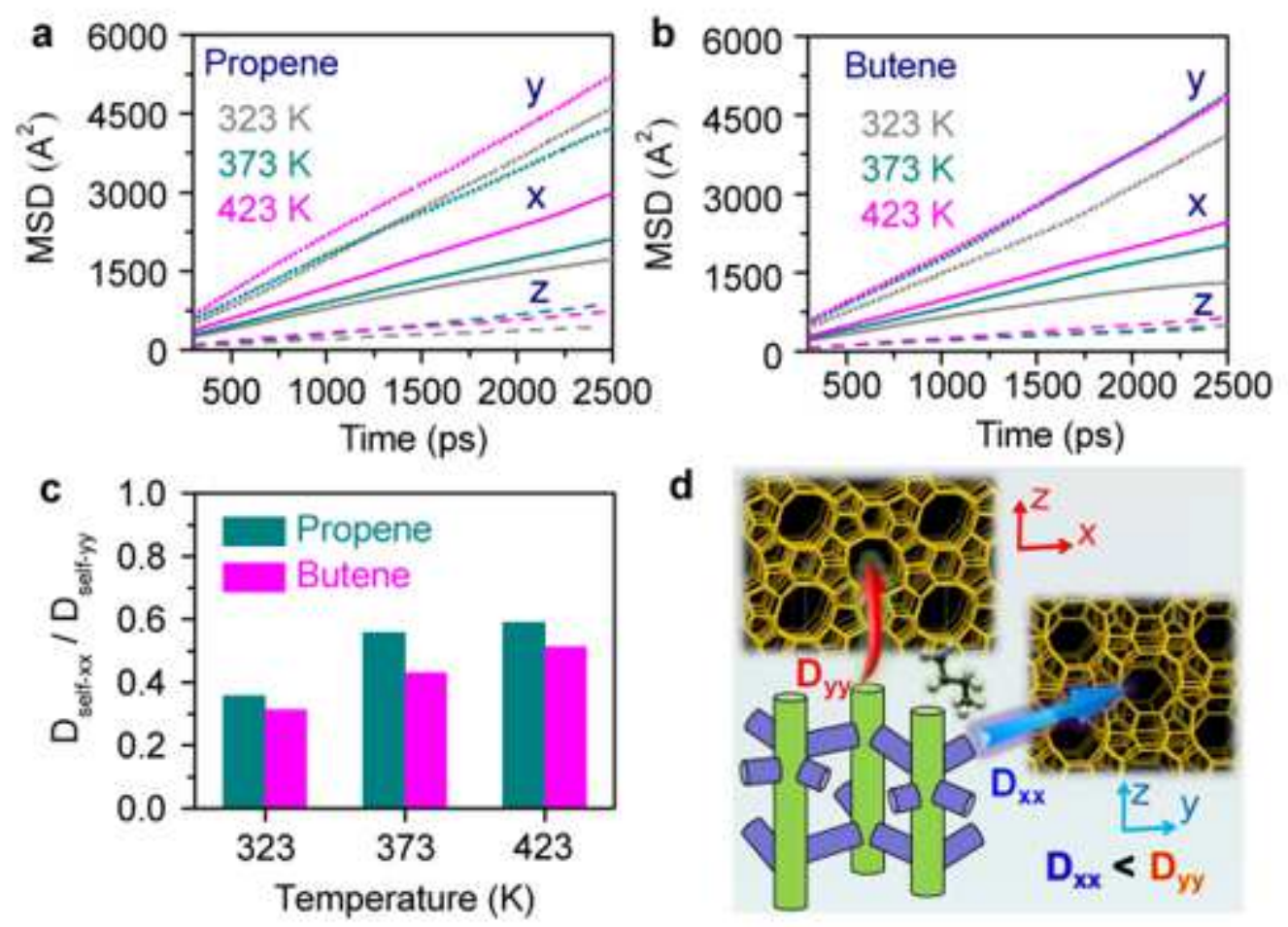

Figure 5

Molecular dynamics simulations on the diffusion of propene and 1-butene molecules in MFI-structured zeolite. a MSDs of propene diffusion in different directions at different temperatures. b MSDs of 1-butene diffusion in different directions at different temperatures. $c$ The ratio of self-diffusion coefficient in $\mathrm{x}$ - and $y$-directions. $d$ Schematic view of olefin molecules diffusion along $x$ - and $y$-directions of MFI type zeolites. The anisotropic components MSDxx, MSDyy and MSDzz curves were calculated by three individual samples, the average MSD, Dself-xx and Dself-yy are reported in this work. 

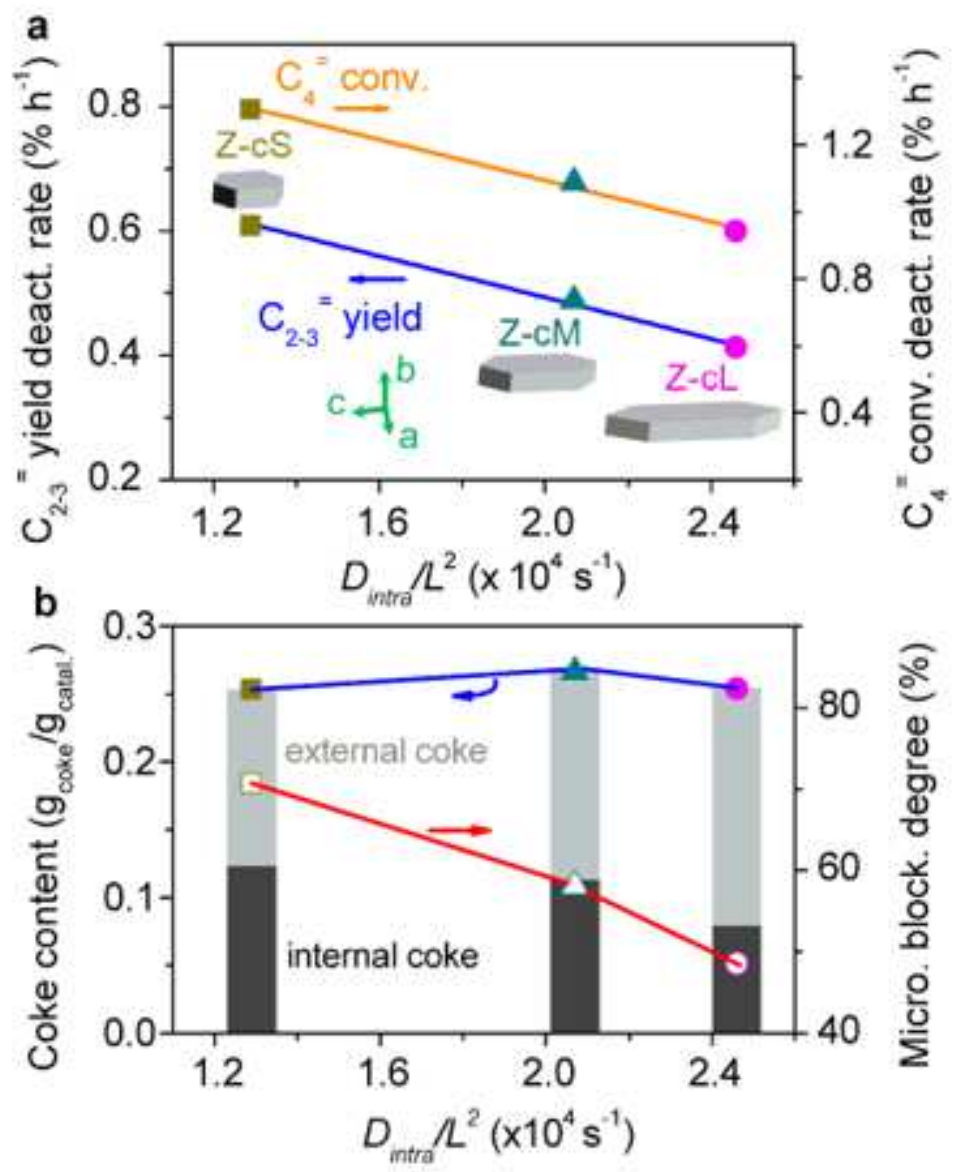

\section{Figure 6}

a Correlation of $\mathrm{C} 4=$ conversions and $\mathrm{C} 2-3=$ yield deactivation rates with the intracrystalline diffusive rates of butene molecules in $\mathrm{H}-\mathrm{ZSM}-5$ crystals. The deactivation rates were calculated on the basis of the catalytic performances displayed in Fig. $2 \mathrm{a}$ and b. The data between $20 \mathrm{~h}$ and $50 \mathrm{~h}$ were chosen due to the actual deactivation time. $\mathrm{b}$ The coke depositions and the micropores blockage degrees after $50 \mathrm{~h}$ of OCC reactions.

\section{Supplementary Files}

This is a list of supplementary files associated with this preprint. Click to download.

- SupplementaryInformationZaikuXie.docx 